\title{
Systemic sclerosis and bilateral lung transplantation: a single centre experience
}

\author{
R. Saggar*,**, D. Khanna ${ }^{\#, * *}$, D.E. Furst ${ }^{\#}$, J.A. Belperio*, G.S. Park ${ }^{\#, \uparrow, ~ S . S . ~ W e i g t *, ~}$ \\ B. Kubak ${ }^{+}$, A. Ardehali ${ }^{\S}$, A. Derhovanessian*, P.J. Clements ${ }^{\#}$, S. Shapiro*, C. Hunter ${ }^{\S}$, \\ A. Gregson ${ }^{+}$, M.C. Fishbein ${ }^{\dagger}$, J.P. Lynch III*, D.J. Ross*** and R. Saggar***
}

ABSTRACT: Lung involvement is the leading cause of death in systemic sclerosis (SSc), but lung transplantation (LT) for systemic disease remains controversial. Our objective was to comprehensively evaluate post-LT outcomes for SSc compared to idiopathic pulmonary fibrosis (IPF).

We retrospectively evaluated bilateral LT recipients (LTRS) with SSc or IPF at our centre between January 1, 2003 and December 31, 2007. The primary end-point was all-cause mortality at $1 \mathrm{yr}$ post-LT. Secondary end-points included assessments of acute rejection (AR), pulmonary function, infection and chronic rejection.

14 patients with SSc and 38 patients with IPF underwent LT. Apart from a younger SSc cohort (53.2 versus 58.8 yrs; $p=0.02$ ), the two groups were well matched. 1 -yr all-cause mortality was no different between SSc $(6.6 \%)$ and IPF (13.1\%) groups, after adjusting for age $(p=0.62)$. Rates of $(A R) \geqslant 2$ were significantly increased for the SSc compared with the IPF group (hazard ratio (HR) 2.91; $p=0.007$ ). Other end-points, including chronic rejection, infection and pulmonary function, showed no differences.

SSc LTRs experience similar survival $1 \mathrm{yr}$ post-LT when compared to IPF. AR rates may be significantly higher in the SSc group. Longer follow-up is necessary to determine the effects of gastrointestinal dysfunction and AR on late allograft function in SSc LTR.

KEYWORDS: Lung transplantation, systemic sclerosis

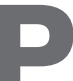

ulmonary arterial hypertension (PAH) and pulmonary fibrosis (PF) are presently the leading causes of systemic sclerosis (SSc)-related death [1]. Lung transplantation (LT) for respiratory insufficiency secondary to systemic disease remains controversial. However, the 2006 International Society of Heart and Lung Transplantation (ISHLT) report deemed LT to be an option for patients with connective tissue disease (CTD), provided the systemic disease was otherwise quiescent [2]. However, this report found the available data to be insufficient to support specific pre-LT guidelines for patients with CTD. Although prior studies of SSc and LT have addressed survival $[1,3,4]$, they may not necessarily have focused on other important post-LT parameters such as rejection, infection and pulmonary function.

The purpose of this manuscript is to comprehensively report our single-centre experience of SSC post-bilateral LT with a focus on rejection, infection and pulmonary function, as well as survival. We use idiopathic pulmonary fibrosis (IPF) as a comparator group, given its similar spectrum of lung disease and prognosis.

\section{STUDY DESIGN AND METHODS}

\section{Study design}

We retrospectively assessed all LT recipients (LTRs) who underwent LT between January 1, 2003 and December 31, 2007 at the University of California, Los Angeles, CA, USA (UCLA). Appropriate consent was obtained in accordance with our institutional review board. We identified all LTRs with SSc (limited or diffuse) based on the American College of Rheumatology criteria per UCLA rheumatology [5]. During this interval, a total of 243 LTs were performed, of which 15 (6.1\%) were done for SSc-associated PF and/or PAH. All LTs for SSc were bilateral, with the exception of one patient who underwent single LT. We also identified all LTRs with IPF who underwent bilateral LT during this period (38 of 243; $15.6 \%$ ). The diagnosis of IPF was established using explant tissue histopathology, defined as usual interstitial pneumonia (UIP) in the appropriate clinical context [6]. SSc explant tissue displayed a mixed histopathology (UIP $(n=9)$; nonspecific interstitial pneumonia (NSIP) with fibrosis $(n=3)$; NSIP without fibrosis $(n=1)$; and features of both UIP and NSIP with fibrosis $(n=2))$.
AFFILIATIONS

${ }^{*}$ Division of Pulmonary and Critical Care Medicine, Dept of Medicine,

\#Division of Rheumatology, Dept of Medicine,

${ }^{+}$Division of Infectious Diseases, Dep of Medicine,

${ }^{\S}$ Division of Cardiothoracic Surgery, Dept of Surgery,

${ }^{f}$ Dept of Pathology and Laboratory Medicine, David Geffen School of Medicine at University of California, Los Angeles, Los Angeles, CA, and "Global Biostatistics and Epidemiology, General Medicine and Inflammation, Amgen Inc., Thousand Oaks, CA, USA

**First and last coauthors

CORRESPONDENCE

R. Saggar

Division of Pulmonary and Critical

Care Medicine

Dept of Medicine

David Geffen School of Medicine at UCLA

Los Angeles

CA 90095-1690

USA

E-mail: rsaggar@mednet.ucla.edu

Received:

Sept 032009

Accepted after revision:

March 122010

First published online:

March 292010 


\section{Patient selection}

Patients with SSc and IPF referred for LT were evaluated in accordance with the general principles established by guidelines for the selection of LT candidates [2].

Potential SSc LTRs were assessed for the extent of gastrooesophageal reflux (GER) and oesophageal/gastrointestinal (GI) dysmotility (collectively, gastrointestinal dysfunction) by evaluation with dual $\mathrm{pH}$ probe study, barium oesophagram, nuclear medicine quantitative gastric emptying study, oesophageal manometry and/or upper endoscopy. SSc LT candidates with objective evidence for GI dysfunction that was unable to be subjectively controlled by aggressive medical therapy (combination of high-dose proton-pump inhibitor and/or $\mathrm{H}_{2}$ blocker, high-dose pro-motility agent and lifestyle modification counselling), were excluded for LT. Importantly, the DeMeester score $[7,8]$ (based on dual $\mathrm{pH}$ probe study) in isolation was neither an inclusion nor exclusion criterion. In addition, potential SSc LTRs were excluded if there was evidence for any one of the following while receiving aggressive medical therapy: symptomatic oesophageal stricture or upper GI ulcer, oesophageal atonia/achalasia or abnormal gastric emptying $(<25 \%$ clearance at 90 min postingestion). Other inclusion and exclusion criteria are outlined in table 1.

\section{Single versus bilateral $L T$}

Preferred bilateral and mandatory single LT are performed for ages $\leqslant 60$ yrs and ages $\geqslant 65$ yrs, respectively. Either single or bilateral LT is possible for ages 61-64 yrs and the following factors determine this decision: obstructive coronary artery disease, degree of $\mathrm{PAH}$, suppurative lung disease, prior thoracic surgery and body habitus. Our programme performs all bilateral LT on cardiopulmonary bypass.

\section{Post-LT immunosuppression and surveillance protocols}

LTR received perioperative immunosuppresion including methylprednisolone, tacrolimus and mycophenolate mofetil, and prophylactic antimicrobials, as previously described [9]. Induction chemotherapy was provided with either basiliximab or rabbit antithymocyte globulin. Surveillance bronchoscopy (including transbronchial biopsies) and bronchoalveolar lavage (BAL) was performed to assess for acute rejection (AR) and possible infection at 1, 3, 6 and 12 months, post-LT. Additional bronchoscopic assessments and/or lower respiratory tract sampling (e.g. sputum) were carried out when clinically indicated and were included in our data collection. Spirometric testing was obtained at surveillance bronchoscopy time points and included per cent reference and absolute values for forced expiratory volume in $1 \mathrm{~s}$ (FEV1), forced vital capacity (FVC) and FEV1/FVC ratio [10].

\section{SSc-specific post-LT prophylaxis}

High-dose proton-pump inhibitors (e.g. pantoprazole $80 \mathrm{mg}$ orally b.i.d.) and pro-motility agents were (re)initiated for SSc LTRs immediately post-LT. An angiotensin-converting enzyme inhibitor was initiated, as tolerated by systemic blood pressure, as prophylaxis for renal crisis. The head of the bed was kept $\geqslant 45^{\circ}$ for GER prophylaxis. Radial arterial catheters were avoided given the high prevalence of Raynaud's phenomenon in SSc.

\section{Study definitions}

PAH was defined by right heart catheterisation (RHC) [11]. An evaluation for other potential causes of PAH [11] was negative in all SSc and IPF cases.

AR was histologically defined using ISHLT criteria [12] and treated as previously described [9]. AR is classified as grade 1 through to grade 4 . An average AR score was calculated by adding the sum of AR grades of each rejection episode during the first year post-LT [13], and dividing by the number of bronchoscopic assessments. Lymphocytic bronchiolitis (LB) was either present or absent.

Chronic rejection clinically manifests as bronchiolitis obliterans syndrome (BOS) and was assessed based on existing ISHLT criteria [14]. BOS was diagnosed after exclusion of AR, infection, increased body mass index and/or anastomotic complications.

Primary graft dysfunction (PGD) was graded post-LT, as previously defined [15].

A lung allocation score (LAS) score was calculated for each patient and used to determine priority for LT [16].

Infection was defined as any microbiological isolate based on either expectorated sputum or BAL fluid (BALF). All BALF was routinely sent for microbiological testing, including for (acid-fast) bacteria, viral and fungal organisms.

\section{Baseline LTR demographics and time-dependent variables}

The baseline demographic and perioperative data included age, sex, renal function, donor/recipient cytomegalovirus (CMV) status, transthoracic echocardiographic estimation of right ventricular systolic pressure, RHC-based haemodynamic variables, LAS, cardiopulmonary bypass time, donor ischaemia time and PGD at $72 \mathrm{~h}$ post LT. The variables analysed between groups over the first year post-LT included spirometry, AR, LB and BOS.

\section{Statistical analysis}

Data were analysed using SAS vs.9.1 (SAS Institute Inc., Cary, NC, USA) and Stata vs.8 (StataCorp LP, College Station, TX, USA). For purposes of this manuscript, the single LT SSc patient data $(n=1)$ was only included in the survival analysis and was intentionally excluded for all other analyses, in an effort to provide homogeneous populations for study comparison. Groups were compared using paired t-test (or Wilcoxon rank-sum tests) for continuous variables, Chi-squared test for categorical variables, and Fisher's exact test for small sample sizes. Descriptive statistics are presented as mean \pm SD or medians (interquartile range). To test for differences within groups over time, paired t-tests and Wilcoxon signed rank tests were performed. As this was an exploratory analysis, there was no adjustment for multiplicity. Significance was prespecified at $\mathrm{p} \leqslant 0.05$. In addition, survival analysis was employed to assess time-to-event outcomes. The Cox proportional hazards model was used to assess differences in time to death post-LT between groups, adjusting for age. The time to first $\mathrm{AR} \geqslant 1$ or $\mathrm{AR} \geqslant 2$, was compared between groups. Kaplan-Meier curves are provided for the survival analysis and time-to-event analysis. 


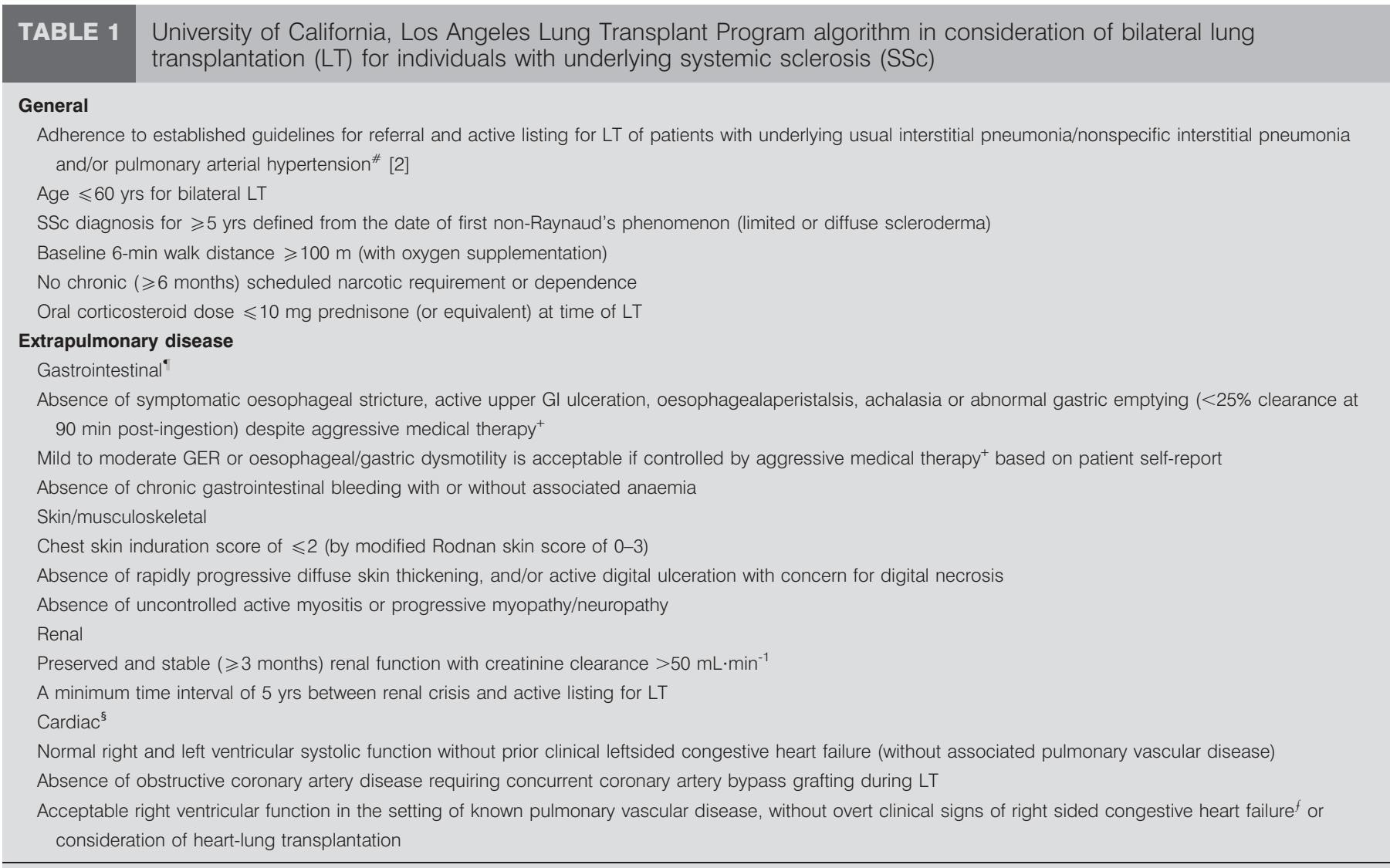

Gl: gastrointestinal; GER: gastro-oesophageal reflux; *: the relatively poorer prognosis of SSc pulmonary arterial hypertension (PAH) compared to idiopathic PAH might warrant earlier referral and active listing for $\mathrm{LT}^{\prime}{ }^{\prime}$ : we recommend the following tests be performed for all potential SSc LT recipients: barium oesophagram, nuclear medicine quantitative gastric emptying study, oesophageal manometry and upper endoscopy; and the following tests considered: dual pH probe study and/or impedance testing; ${ }^{+}$: combination of high-dose proton-pump inhibitor and/or $\mathrm{H}_{2}$ blocker, high-dose pro-motility agent and antireflux measures, including lifestyle modification counselling; ${ }^{s}$ : consider cardiac magnetic resonance imaging; ${ }^{f}$ : absence of clinical congestive heart failure, and preservation of liver function testing (including synthetic function).

\section{RESULTS}

A total of 243 LTs were performed between January 1, 2003 and December 21, 2007. 14 and 38 bilateral LTs were performed in patients with SSc and IPF, respectively. Among SSc LTRs, the indication for LT was isolated PAH in two patients (14\%), PF in six patients $(43 \%)$ and combined $\mathrm{PAH} / \mathrm{PF}$ in six patients $(43 \%)$; $16(42 \%)$ of 38 IPF patients had associated PAH. The pre-LT median (interquartile range) pulmonary arterial (PA) pressure by RHC was similar between the SSc and IPF groups 29 (23-45) versus 23.5 (20-32.5) $\mathrm{mmHg}$, respectively; $\mathrm{p}=0.11$. There were no other significant differences in pre-LT haemodynamic parameters between groups (table 2). The median DeMeester score in our SSc cohort was 42.6, suggesting pathologic GER despite aggressive medical therapy (table 2). A DeMeester score was not routinely performed in the IPF group.

\section{Baseline characteristics}

Demographics were similar between the groups (table 2). However, the mean age of LTRs with SSc was younger (53.2 versus 58.8 yrs; $\mathrm{p}=0.02)$, and 11 of $14(79 \%)$ had limited disease. Median (interquartile range) follow-up was 632 (442-1033) and
788 (550-950) days for patients with SSc and IPF, respectively $(p=0.99)$. The pre-LT creatinine clearance for patients with SSc was within normal limits: $92.2(64.8-118) \mathrm{mL} \cdot \mathrm{min}^{-1}$ (table 2). There were no differences between groups in the rates of preLT human leukocyte antigen mismatching or the type of induction therapy (data not shown). Pre-LT spirometry was similar between both SSc and IPF (table 3) with the sole exception of FEV1/FVC ratio, which was significantly decreased in the SSc group. It should be noted that SSc serological data, specifically with regards to autoantibodies (i.e. anti-centromere and anti-topoisomerase, etc.), was not uniformly performed in our SSc cohort.

\section{Survival analysis}

During the first year post-LT, one (6.6\%) SSc and five (13.1\%) IPF LTRs died $(\mathrm{p}=0.62)$. During a median range follow-up of $632(0-1,474)$ days (SSc) and 788 days (IPF), there were three $(21.4 \%)$ deaths in patients with SSc and 11 (28.9\%) deaths in patients with IPF $(p=0.69)$ (fig. 1). In the SSc group, causes of death included complications of BOS $(n=1)$, scleroderma renal crisis $(n=1)$ and complications of induction anaesthesia in the 
TABLE 2 Pre-transplant demographics and post-transplant outcomes at 1 year

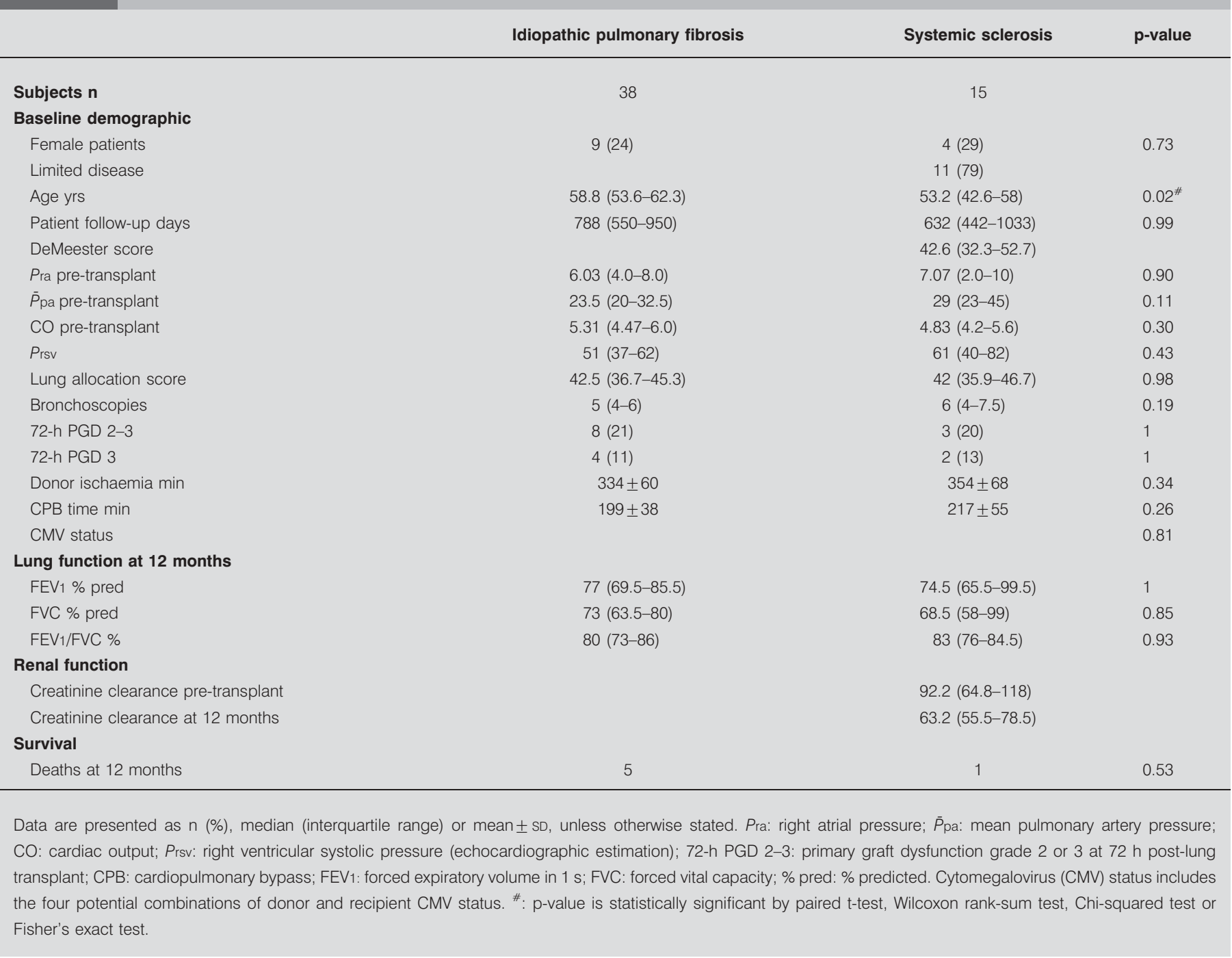

setting of severe PAH $(n=1)$. In comparison, causes of death for IPF included complications of BOS $(n=3)$, sepsis / pneumonia $(n=4)$, community-acquired adenoviral infection $(n=1)$, perforated bowel $(n=1)$, air embolus $(n=1)$ and complications of thorocosternotomy wound dehiscence $(n=1)$. Among those who died during the study period, the median (range) time followed was 481 (1-612) days in the SSc group, compared with 233 (38$390)$ days in the IPF group $(p=0.64)$.

\section{TABLE 3 Pre-transplant pulmonary function}

\begin{tabular}{|c|c|c|c|}
\hline Pre-transplant spirometric parameter & Systemic sclerosis & Idiopathic pulmonary fibrosis & p-value \\
\hline $\mathrm{FEV}_{1} \mathrm{~L}$ & $1.5 \pm 0.6$ & $1.7 \pm 0.8$ & 0.26 \\
\hline FEV $1 \%$ pred & $47.3 \pm 19.8$ & $51.4 \pm 16.9$ & 0.45 \\
\hline FVC L & $1.7 \pm 0.7$ & $1.9 \pm 1.0$ & 0.48 \\
\hline FVC \% pred & $42.2 \pm 17.3$ & $49.3 \pm 18.3$ & 0.20 \\
\hline FEV $1 / F V C$ & $86.3 \pm 6.5$ & $92.7 \pm 15.7$ & $0.04^{\#}$ \\
\hline$D \mathrm{~L}, \mathrm{CO} \mathrm{mL} \cdot \mathrm{min}^{-1} \cdot \mathrm{mmHg}^{-1}$ & $6.7 \pm 1.9$ & $7.3 \pm 3.6$ & 0.66 \\
\hline$D L$, co \% pred & $27.9 \pm 9.0$ & $27.5 \pm 11.4$ & 0.91 \\
\hline
\end{tabular}

Data are presented as mean \pm SD, unless otherwise stated. FEV1: forced expiratory volume in $1 \mathrm{~s}$; \% pred: \% predicted; FVC: forced vital capacity; DL,CO: diffusing capacity of the lung for carbon monoxide. ${ }^{*}$ : p-value is statistically significant by paired t-test or Wilcoxon rank-sum test. 


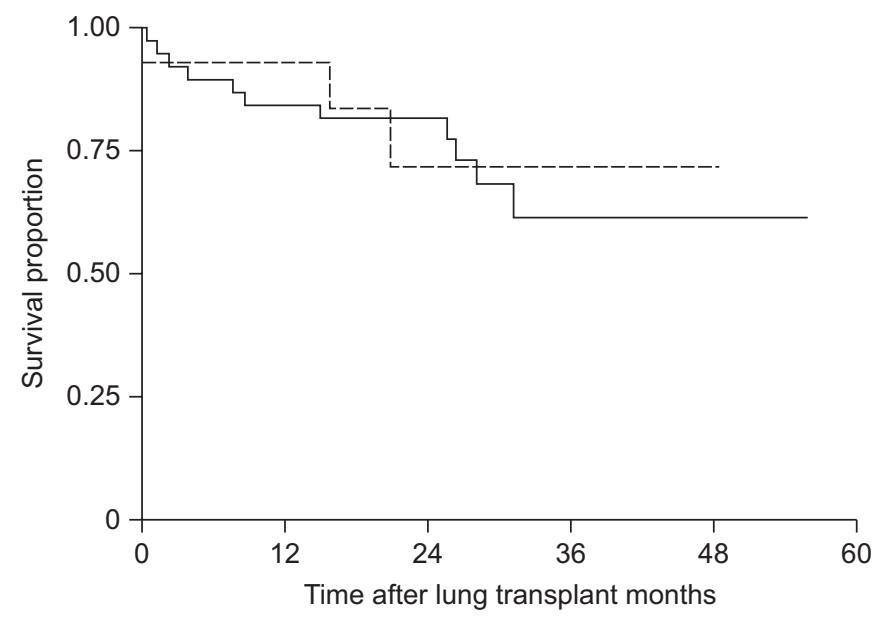

FIGURE 1. Kaplan-Meier plot displaying the survival estimates for the idiopathic pulmonary fibrosis group (-) versus the systemic sclerosis group $(----)$. There was no significant difference in overall survival between groups (logrank test, $p=0.69$ ).

\section{Infection}

During the first year post-LT, there were no significant differences between mean \pm SD number of BALF $(5.7 \pm 1.9$ versus $4.8 \pm 2.3 ; \mathrm{p}=0.19)$ or sputum $(3.3 \pm 3.5$ versus $4.0 \pm 4.8$; $\mathrm{p}=0.65$ ) assessments made between LTRs with SSc and IPF, respectively. In addition, no differences were observed between the median number of bacterial, viral (including $\mathrm{CMV}$ ), fungal or acid-fast bacilli isolates (table 4).

\section{Acute rejection}

During the first year post-LT, the proportion of patients who developed $A R \geqslant 1$ was higher in the SSc group (nine out of 13; $69 \%$ ) compared to the IPF group (14 out of $37 ; 38 \%$ ), a difference which trended toward statistical significance $(p=0.06$; fig. 2$)$. In addition, the proportion of patients that developed AR scores $\geqslant 2$ was significantly higher in the SSc group (eight out of $13 ; 62 \%$ ) compared with the IPF group (eight out of $37 ; 22 \%$ ) (hazard ratio (HR) 2.91; $p=0.007$.) (fig. 3). After adjusting for age, this finding remained significant $(p=0.049)$. The median (interquartile range) time to first episode of AR score $\geqslant 2$ was 75.0 (35.5-103.0) in SSc $(n=8)$ versus $43.0(20.5-186.5)$ days in $\operatorname{IPF}(n=8) \quad(p=0.49)$. The average AR score during the first year post-LT was significantly higher in the SSc group, $0.46 \pm 0.44$ compared with $0.22 \pm 0.36$ in the IPF group $(p=0.048)$ (table 4$)$. However, the proportion of SSc and IPF LTRs with LB (11 out of 13 (85\%) versus 24 out of $37(65 \%) ; p=0.29)$, and the median number of bronchoscopic assessments during the first year post-LT were similar between both groups (4.5 (3.0-6.0) versus 4.0 (2.0-5.0); $\mathrm{p}=0.78)$. The number of SSc and IPF patients who developed AR grade 1, 2 or 3 were one $(8 \%)$, three $(23 \%)$ and five $(38 \%)$, compared with six (16\%), four $(11 \%)$ and four $(11 \%)$, respectively (table 4$)$.

\section{Pulmonary function}

At $1 \mathrm{yr}$ post-LT, there were no significant differences in median values for FEV1 (74.5 versus $77.0 \%$ pred), FVC (68.5 versus $73.0 \%$ pred) or FEV1/FVC ratio (83.0 versus $80.0 \%$ ),

\begin{tabular}{lccc} 
TABLE 4 & $\begin{array}{c}\text { Post-transplant infection and rejection at } 1 \text { year } \\
\text { Idiopathic pulmonary } \\
\text { fibrosis }\end{array}$ & $\begin{array}{c}\text { Systemic } \\
\text { sclerosis }\end{array}$ & p-value \\
\hline Subjects $\mathbf{n}$ & 37 & 13 & \\
Infections at & & & \\
$\mathbf{1 2}$ months $^{\#}$ & & & \\
Bacteria & $0.17(0-0.5)$ & $0.2(0-0.5)$ & 0.54 \\
Fungus & $0(0-0.25)$ & $0(0-0.25)$ & 0.39 \\
Virus & $0(0-0)$ & $0(0-0)$ & 0.91 \\
AFB & $0(0-0)$ & $0(0-0.13)$ & 0.22 \\
Rejection (TBBx at & & & \\
12 months) & & 69 & 0.06 \\
AR \% & 38 & 31 & $0.05^{\circ}$ \\
Never rejection \% & 62.2 & $0.5(0-0.67)$ & $0.05^{\circ}$ \\
Average AR score & $0(0-0.4)$ & $11(85)$ & 0.29 \\
LB & $24(65)$ & 62 & $0.01^{\circ}$ \\
AR score $\geqslant 2 \%$ & 22 &
\end{tabular}

Data are presented as $\mathrm{n}(\%)$ or median (interquartile range), unless otherwise stated. AFB: acid-fast bacilli; TBBx: transbronchial biopsy; AR: acute rejection; LB: lymphocytic bronchitis. \#: accounts for bronchoalveolar lavage and expectorated sputum sampling; " $\mathrm{p}$-value is statistically significant by paired t-test, Wilcoxon rank-sum test, Chi-squared test or Fisher's exact test.

when comparing SSc to IPF, respectively (table 2). In addition, no differences were found between groups when comparing the change in these same spirometric parameters between 3 and 6 months, 6 and 12 months, and 3 and 12 months, post-LT (data not shown).

\section{Chronic rejection (BOS)}

The median time to onset of BOS (1,492 versus 1,322 days) and freedom from BOS at 1 ( $70 \%$ versus $88 \%$ ), 2 (63\% versus $84 \%$ ) and $3(52 \%$ versus $60 \%)$ yrs post-LT were similar between the SSc and IPF groups, respectively (Mantel-Cox, $\mathrm{p}=0.29$ ) (fig. 4).

\section{DISCUSSION}

Our report marks the largest cohort of SSc patients who underwent bilateral LT at a single centre. The primary endpoint of all-cause mortality at $1 \mathrm{yr}$ post-LT was similar between the SSc $(n=1 ; 6.6 \%)$ and IPF $(n=5 ; 13.1 \%)$ groups. In addition, during a median (range) follow-up of 632 (0$1,474)$ days for the SSc group and $788(12-1,700)$ days for the IPF group, the post-LT survival (78.6\% versus $71.1 \%$ ) was no different. $A R \geqslant 2$ was significantly increased in the SSc group $(62 \%)$ compared with the IPF group (22\%), over the initial $1 \mathrm{yr}$ post-LT. Nonetheless, the freedom from BOS was similar in both groups, and there were no differences in pulmonary function or rates of infection.

Similar to prior reports, we chose IPF as a comparator group given the similar spectrum of lung disease to SSc. In addition, IPF uniquely represents the most common indication for LT at our centre. At baseline, the two groups showed no significant differences with the exception of a statistically lower mean age at time of LT in the SSc group (53.2 versus 58.8 yrs). While there was no predefined selection for PAH or limited SSc in our cohort, $86 \%$ of the SSc patients had PAH as an element of their 


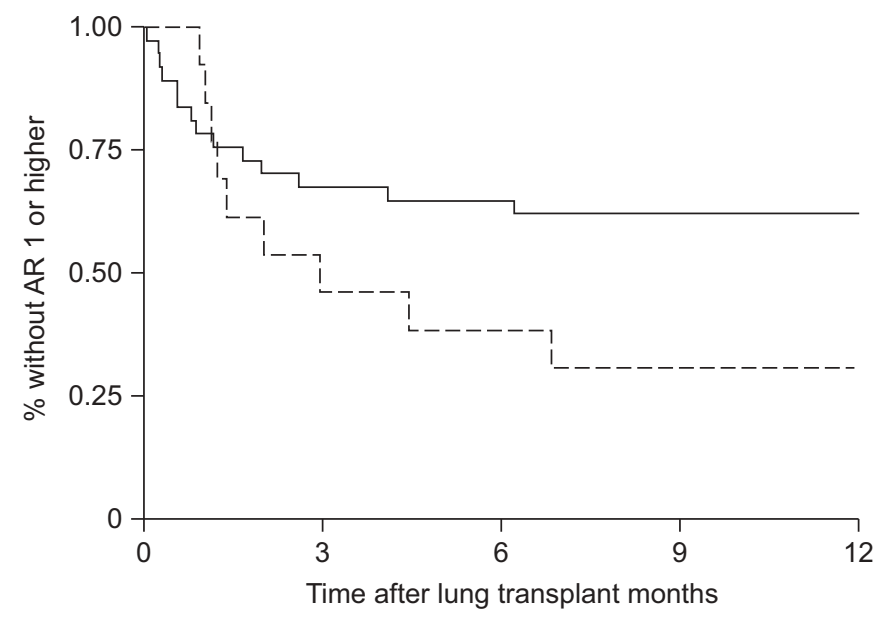

FIGURE 2. Kaplan-Meier plot displaying the estimated time to first acute rejection $\geqslant 1(A R \geqslant 1)$ for the idiopathic pulmonary fibrosis group (-) versus the systemic sclerosis group (-- - ), during the first year after lung transplantation. There is no significant difference between groups (log-rank test, $p=0.06$ ).

disease and $79 \%$ had limited disease. We suspect limited disease was over-represented given the inherent early mortality of individuals with diffuse disease [17].

The largest retrospective case series to date reviewed $47 \mathrm{SSc}$ LTs in over 23 US centres. The overall 1-yr survival was 68\%; however, the results must be interpreted in the context of variable transplant experience, and lack of standardised selection criteria [3]. Recently, ScHACHNA et al. [1] reported survival rates post-LT in SSc $(n=29)$ compared to either IPF $(n=70)$ or idiopathic PAH $(n=38)$, at two large university centres [1]. The majority of LTs performed in all three cohorts were single LT and the 2 -yr cumulative survival was $>60 \%$ and similar across all three groups. Importantly, these authors focused on pre-LT clinical parameters, demographics and overall survival, and less so on other accepted predictors of post-LT survival, including AR, infection and BOS [18].

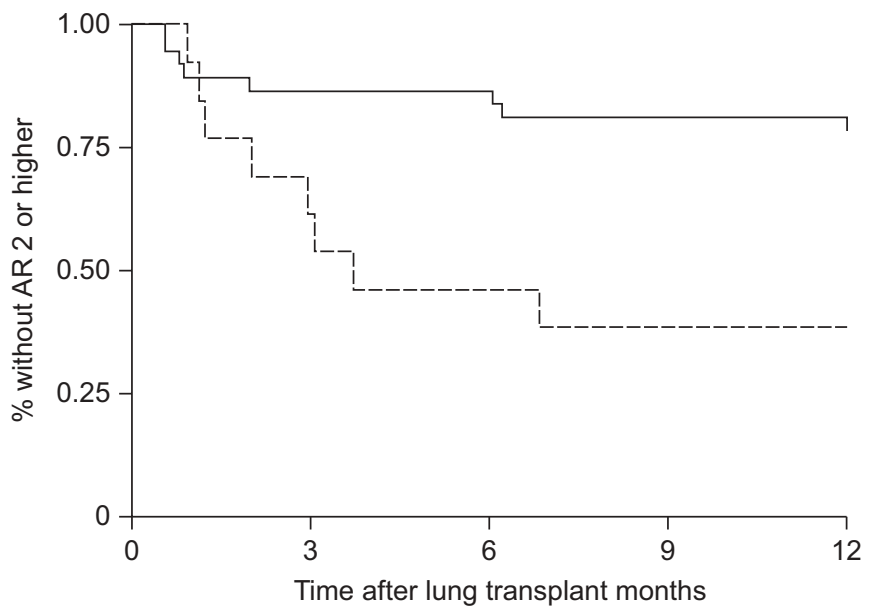

FIGURE 3. Kaplan-Meier plot displaying the estimated time to first acute rejection $\geqslant 2(A R \geqslant 2)$ for the idiopathic pulmonary fibrosis group (-) versus the systemic sclerosis group $(----)$, during the first year after lung transplantation. There is a significant difference between groups (log-rank test, $p=0.007$ ).

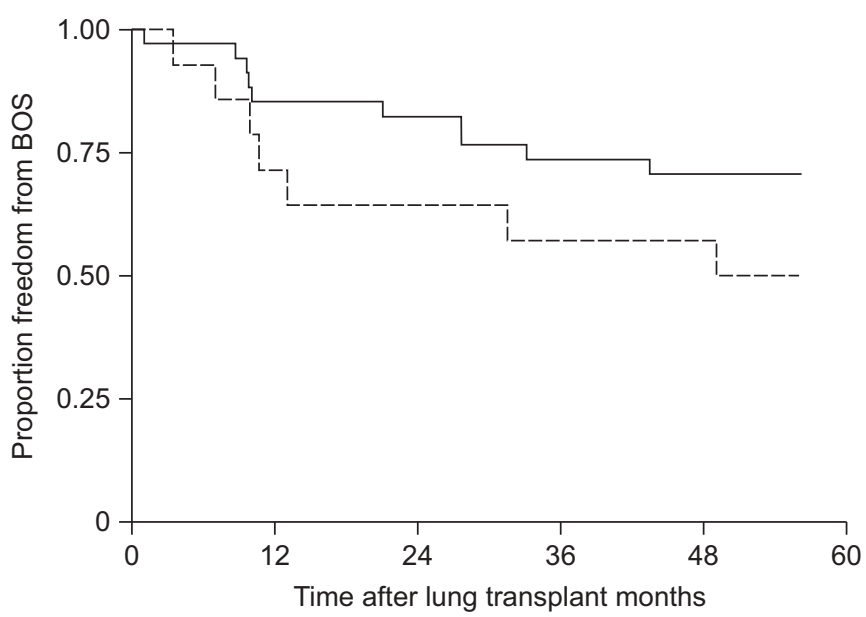

FIGURE 4. Kaplan-Meier plot displaying freedom from chronic rejection bronchiolitis obliterans syndrome (BOS) post-lung transplantation for the idiopathic pulmonary fibrosis group (-) versus the systemic sclerosis group $(----)$. For the first 5 yrs after lung transplantation, there is no significant difference between the two groups (log-rank, $p=0.14$ ).

In our study, 1-yr post-LT survival tended to be greater among SSc patients with a survival rate of $93.4 \%$, compared with $86.9 \%$ for IPF. In addition, over a median follow-up period of $\sim 2$ yrs, the cumulative survival rate was similar between SSc $(80 \%)$ and IPF $(71.1 \%)$. Of the deaths that occurred in our SSc group $(n=3)$, one patient died within $24 \mathrm{~h}$ of LT due to complications of general anaesthesia in the setting of severe $\mathrm{PAH}$ and right ventricular dysfunction. After this unexpected death, we instituted a policy to perform RHC just prior to anaesthesia induction in patients with significant pre-LT PAH, in order to optimise pulmonary haemodynamics. The second patient died after 481 days due to renal crisis, while the third patient died after 635 days secondary to complications of BOS. Although there was no difference in mortality comparing nonPAH with PAH patients or limited versus diffuse SSc patients (data not shown), future studies with larger numbers should incorporate comparison of these groups.

LT for CTD remains controversial, due to the unpredictable capacity for systemic organ involvement and potential for disease recurrence. One of the SSc deaths was in fact due to renal crisis, a well-known complication of SSc. To date, there has been no report to support recurrence of SSc in the lung allograft.

We observed a statistically increased proportion of patients with SSc developing AR $\geqslant 2$ compared with the IPF group $(p=0.007)$. With the exception of age, other known predictors of post-LT morbidity (i.e. recipient PAH, donor ischaemia time, infection and PGD) were similar between groups and probably do not explain this finding. We hypothesise that the inherent predisposition for GI dysfunction (particularly oesophageal dysmotility) may contribute to the increased AR seen in our SSc cohort. However, the absence of a systematic assessment for GER in the IPF (pre- and post-LT) and SSc (post-LT) groups makes it difficult to draw any definitive conclusion regarding the association of GI dysfunction and post-LT alloreactivity. To test this hypothesis in a subsequent study, researchers may consider an assessment of BALF pepsin [19] and/or bile acids, incorporation of impedance testing to evaluate for non-acid 
reflux [20], the contribution of the actual surgical intervention [21], and the effect of azithromycin use [22]. Nevertheless, it is reassuring that, despite the evidence of widespread GER in our SSc patients (median DeMeester score 42.6), patient survival was not affected for a median follow-up of $\sim 2$ yrs.

The frequency and severity of AR post-LT is a reproducible risk factor for subsequent BOS. In turn, BOS is the single most important determinant of late mortality ( $>1 \mathrm{yr}$ ) post-LT [23]. Although we found an increased rate of AR in our SSc cohort, freedom from BOS was no different at 1 (70\% versus $88 \%), 2$ (63\% versus $84 \%$ ), and 3 (52\% versus $60 \%$ ) yrs post-LT, when compared with IPF. The proportion of IPF LTRs with freedom from BOS is consistent with the reported ISHLT registry data of $75 \%$ at 2.5 yrs [23]. Importantly, severe PGD at $72 \mathrm{~h}$ post-LT is another accepted risk factor for subsequent BOS [24] and, apart from showing no difference between groups, the $13 \%$ PGD rate in SSc is consistent with a recent large registry report [25]. Interestingly, freedom from BOS for SSc (compared with IPF) appeared decreased at all time points, but was not statistically significant. Longer follow-up is necessary to draw any conclusions regarding freedom from BOS for SSc LTRs.

Based on our experience and that of other investigators [1, 3, 4, 26], and in concert with accepted guidelines for the selection of LT candidates [27], we have suggested a UCLA algorithm for the assessment of patients with SSc for LT (table 1). We recommend the pre-LT evaluation be a coordinated, multidisciplinary effort to avoid post-LT morbidity and mortality related to the potential for extrapulmonary disease in the setting of underlying SSc. The evaluation for GI dysfunction is particularly important given the association between GER and chronic rejection (BOS) post-LT [28], justifying the increasing consideration of early anti-reflux surgery [26, 29-31]. Importantly, none of our SSc patients have undergone anti-reflux surgery to date.

Our study has limitations, especially those pertaining to a retrospective analysis including inherent selection bias and the potential for missing data. We recognise our limited sample size and available longitudinal follow-up, rendering it difficult to make a definitive conclusion regarding survival. In addition, we did not systematically collect SSc serologic autoantibody data and as such, were unable to report this baseline demographic. To our knowledge, this manuscript represents the largest single centre experience of bilateral LTs for SSc published to date; moreover, we have conducted an additional six bilateral LT for SSc since January 1, 2008. This is the first study to comprehensively report the post-bilateral LT complications at a single centre in patients with SSc including acute and chronic rejection. Importantly, all SSc LTs at our institution to date, with one exception, have been bilateral, a direct consequence of our centre's donor protocol, which is largely driven by recipient age. These limitations not withstanding, we demonstrate that cumulative survival of SSc at 1 and 2 yrs was similar to those with IPF, with an overall survival of $80 \%$ during a median follow-up of 632 days. However, there may be an increased risk of AR in SSc LTR, and whether this translates to an increased risk of future BOS requires further study.

\section{SUPPORT STATEMENT}

D. Khanna was supported by a National Institutes of Health Award (NIAMS K23 AR053858-03) and the Scleroderma Foundation (New Investigator Award).

\section{STATEMENT OF INTEREST}

Statements of interest for D.E. Furst and G.S. Park can be found at www.erj.ersjournals.com/misc/statements.dtl

\section{REFERENCES}

1 Schachna L, Medsger TA Jr, Dauber JH, et al. Lung transplantation in scleroderma compared with idiopathic pulmonary fibrosis and idiopathic pulmonary arterial hypertension. Arthritis Rheum 2006; 54: 3954-3961.

2 Orens JB, Estenne M, Arcasoy S, et al. International guidelines for the selection of lung transplant candidates: 2006 update-a consensus report from the Pulmonary Scientific Council of the International Society for Heart and Lung Transplantation. J Heart Lung Transplant 2006; 25: 745-755.

3 Massad MG, Powell CR, Kpodonu J, et al. Outcomes of lung transplantation in patients with scleroderma. World J Surg 2005; 29: 1510-1515.

4 Levine SM, Anzueto A, Peters JI, et al. Single lung transplantation in patients with systemic disease. Chest 1994; 105: 837-841.

5 Masi, AT. Preliminary criteria for the classification of systemic sclerosis (scleroderma). Arthritis Rheum 1980; 23: 581-590.

6 American Thoracic Society/European Respiratory Society International Multidisciplinary Consensus Classification of the Idiopathic Interstitial Pneumonias. This joint statement of the American Thoracic Society (ATS), and the European Respiratory Society (ERS) was adopted by the ATS board of directors, June 2001 and by the ERS Executive Committee, June 2001. Am J Respir Crit Care Med 2002; 165: 277-304.

7 Johnsson F, Joelsson B, Isberg PE. Ambulatory 24 hour intraesophageal $\mathrm{pH}$-monitoring in the diagnosis of gastroesophageal reflux disease. Gut 1987; 28: 1145-1150.

8 Schindlbeck NE, Heinrich C, Konig A, et al. Optimal thresholds, sensitivity, and specificity of long-term $\mathrm{pH}$-metry for the detection of gastroesophageal reflux disease. Gastroenterology 1987; 93: 85-90.

9 Weigt SS, Elashoff RM, Huang C, et al. Aspergillus colonization of the lung allograft is a risk factor for bronchiolitis obliterans syndrome. Am J Transplant 2009; 9: 1903-1911.

10 Hankinson JL, Odencrantz JR, Fedan KB. Spirometric reference values from a sample of the general U.S. population. Am J Respir Crit Care Med 1999; 159: 179-187.

11 Simonneau G, Galie N, Rubin LJ, et al. Clinical classification of pulmonary hypertension. J Am Coll Cardiol 2004; 43: 5S-12S.

12 Trulock EP, Edwards LB, Taylor DO, et al. Registry of the International Society for Heart and Lung Transplantation: twentysecond official adult lung and heart-lung transplant report-2005 J Heart Lung Transplant 2005; 24: 956-967.

13 Girgis RE, Tu I, Berry GJ, et al. Risk factors for the development of obliterative bronchiolitis after lung transplantation. J Heart Lung Transplant 1996; 15: 1200-1208.

14 Estenne M, Maurer JR, Boehler A, et al. Bronchiolitis obliterans syndrome 2001: an update of the diagnostic criteria. J Heart Lung Transplant 2002; 21: 297-310.

15 Christie JD, Carby M, Bag R, et al. Report of the ISHLT Working Group on Primary Lung Graft Dysfunction part II: definition. A consensus statement of the International Society for Heart and Lung Transplantation. J Heart Lung Transplant 2005; 24: 1454-1459.

16 United network for organ sharing. www.unos.org/resources Date last accessed: January, 2010. Date last updated: March, 2010.

17 Jacobsen S, Ullman S, Shen GQ, et al. Influence of clinical features, serum antinuclear antibodies, and lung function on survival of patients with systemic sclerosis. J Rheumatol 2001; 28: 2454-2459.

18 Belperio JA, Weigt SS, Fishbein MC, et al. Chronic lung allograft rejection: mechanisms and therapy. Proc Am Thorac Soc 2009; 6: 108-121. 
19 Stovold R, Forrest IA, Corris PA, et al. Pepsin, a biomarker of gastric aspiration in lung allografts: a putative association with rejection. Am J Respir Crit Care Med 2007; 175: 1298-1303.

20 Savarino E, Bazzica M, Zentilin P, et al. Gastroesophageal reflux and pulmonary fibrosis in scleroderma: a study using $\mathrm{pH}$-impedance monitoring. Am J Respir Crit Care Med 2009; 179: 408-413.

21 Young LR, Hadjiliadis D, Davis RD, et al. Lung transplantation exacerbates gastroesophageal reflux disease. Chest 2003; 124: 1689-1693.

22 von Rosensteil NA, Adam D. Macrolide antibacterials. Drug interactions of clinical significance. Drug Saf 1995; 13: 105-122.

23 Trulock EP, Christie JD, Edwards LB, et al. Registry of the International Society for Heart and Lung Transplantation: twentyfourth official adult lung and heart-lung transplantation report2007. J Heart Lung Transplant 2007; 26: 782-795.

24 Huang HJ, Yusen RD, Meyers BF, et al. Late primary graft dysfunction after lung transplantation and bronchiolitis obliterans syndrome. Am J Transplant 2008; 8: 2454-2462.

25 Kuntz CL, Hadjiliadis D, Ahya VN, et al. Risk factors for early primary graft dysfunction after lung transplantation: a registry study. Clin Transplant 2009; 23: 819-830.
26 Gasper WJ, Sweet MP, Golden JA, et al. Lung transplantation in patients with connective tissue disorders and esophageal dysmotility. Dis Esophagus 2008; 21: 650-655.

27 Maurer JR, Frost AE, Estenne M, et al. International guidelines for the selection of lung transplant candidates. The International Society for Heart and Lung Transplantation, the American Thoracic Society, the American Society of Transplant Physicians, the European Respiratory Society. J Heart Lung Transplant 1998; 17: 703-709.

28 Hadjiliadis D, Duane Davis R, Steele MP, et al. Gastroesophageal reflux disease in lung transplant recipients. Clin Transplant 2003; 17: 363-368.

29 Cantu E 3rd, Appel JZ 3rd, Hartwig MG, et al. J. Maxwell Chamberlain Memorial Paper. Early fundoplication prevents chronic allograft dysfunction in patients with gastroesophageal reflux disease. Ann Thorac Surg 2004; 78: 1142-1151.

30 Patti MG, Gasper WJ, Fisichella PM, et al. Gastroesophageal reflux disease and connective tissue disorders: pathophysiology and implications for treatment. J Gastrointest Surg 2008; 12: 1900-1906.

31 Shitrit D, Amital A, Peled N, et al. Lung transplantation in patients with scleroderma: case series, review of the literature, and criteria for transplantation. Clin Transplant 2009; 23: 178-183. 